\title{
LOS HUERTOS FAMILIARES TRADICIONALES SOPORTE DE SEGURIDAD ALIMENTARIA EN COMUNIDADES CAMPESINAS DEL ESTADO DE MORELOS, MÉXICO
}

\section{Traditional Family Orchards as a Means of Alimentary Security In Rural Communities of the State of Morelos, Mexico}

\author{
Rafael Monroy-Martínez¹, Alma Ponce-Díaz¹, Hortensia Colín-Bahena1, \\ Columba Monroy-Ortiz ${ }^{1}$, Alejandro García-Flores ${ }^{{ }^{*}}$
}

${ }^{1}$ Cuerpo Académico de Unidades Productivas Tradicionales, Laboratorio de Ecología, Centro de Investigaciones Biológicas, Universidad Autónoma del Estado de Morelos, México.

E-mail: *alejandro.garcia@uaem.mx

\section{Resumen}

Los huertos familiares tradicionales, son unidades productivas de las comunidades campesinas del Estado de Morelos, México, las cuales se caracterizan por su riqueza de especies frutales, cuyo manejo está determinado por el significado cultural local de las especies. La estructura ecológica es resultado del conocimiento social que asegura la producción de alimentos y la escasez de agua. El objetivo fue analizar la importancia de los huertos familiares tradicionales en la seguridad alimentaria de la comunidad de Pueblo Nuevo, Tlaltizapan, Morelos, México. Para su contrastación se emplearon técnicas ecológicas y etnobotánicas. La riqueza de plantas fue de 45 especies y dos variedades, con uso múltiple y de tres animales domésticos alimentarios, dos de compañía y uno de carga. La escases de agua extrema se resuelve de tres formas, 1 . administrando con autonomía social su abasto y distribución, 2. el agua de los servicios de limpieza se reutiliza y 3. Se capta agua de lluvia. La producción sostenida de alimentos vegetales y animales domésticos y el destino para autoabasto permite la seguridad alimentaria de las comunidades campesinas de Morelos, México.

Palabras claves: Conocimiento, Aprovechamiento, Unidades productivas, Diversidad biocultural.

\begin{abstract}
Traditional family orchards are productive units of the peasant communities in the State of Morelos, Mexico, which are characterized by the richness of their tree fruit species, whose management is determined by the cultural significance of local species. The ecological structure is a result of social knowledge that ensures the production of food and tackles water shortages. The objective was to analyze the importance of traditional family orchards in the food security of the community of Pueblo Nuevo, Tlaltizapan, Morelos, Mexico. To contrast between results, both ethno-botanical and ecological techniques were applied. The richness of plants was of 45 species and two varieties with multiple uses and of domestic animals of three used as foodstuff, two as pets and one as pack animal. The extreme shortage of water is resolved in three ways, 1 . Managing with social autonomy its supply and distribution, 2. Reusing water used for cleaning services and 3 . Collectin grain water. The sustained production of plant food stuffs and domestic animals, and their role in self-consumption, guarantee the food security of the rural communities of Morelos, Mexico.
\end{abstract}

Keywords: Knowledge, Resource uses, Productive units, Biocultural diversity. 


\section{INTRODUCCIÓN}

La diversidad biológica de México está vinculada a la seguridad alimentaria, porque ha permitido que la mayoría de los grupos sociales tengan acceso físico, social y económico a los alimentos nutritivos para satisfacer sus necesidades (FAO 2012).

No obstante, las estructuras de gobernanza son inadecuadas para asegurar la transparencia y la no discriminación de los grupos vulnerables.Esto lleva por un lado, al deterioro de la seguridad social como factor esencial que agrava el hambre y la inseguridad alimentaria. Por otro, la falta del compromiso político en la consideración de la lucha contra el hambre y la malnutrición como prioridad, incluye la incapacidad de cumplir las promesas de las autoridades electas (CDESC 1999).

Las políticas públicas y el establecimiento de prioridades respecto a los planes, programas y mecanismos de financiamiento para hacer frente al hambre, la malnutrición y la inseguridad alimentaria, carecen de coherencia, porque no prestan atención especial a las poblaciones vulnerables que padecen incertidumbre alimentaria, consecuente los servicios estatales son mínimos en las zonas campesinas e indígenas cancelando, además, la consulta de representantes de las comunidades en los procesos de toma de decisiones que afecten a sus medios de producción locales y los saberes que los soportan (Balvanera \& Cotler 2009).

La cooperación y financiamiento fragmentados, la dispersión de la ayuda en proyectos que carecen de viabilidad para tener repercusiones significativas que incrementan los costos de administración. Además, la pobreza y el acceso inadecuado a los alimentos, derivan de las elevadas tasas de desempleo junto con la distribución desigual de los recursos productivos como la tierra, el agua, el crédito, el conocimiento, salarios bajos, la población rural y urbana marginal cancelan el crecimiento de la producción agrícola tradicional.

A la vez, la ausencia de un sistema comercial multilateral abierto, no discriminatorio, equitativo, sin distorsiones y transparente que impulse la agricultura tradicional y el desarrollo rural generan inseguridad de la tenencia de la tierra y del acceso a la misma, el agua y otros recursos bioculturales.

La inversión internacional y nacional ignora la producción de la ganadería de traspatio en los agroecosistemas tradicionales, en especial para los pequeños productores quienes no tienen acceso a las tecnologías, insumos e instituciones.
La expansión histórica del área metropolitana de la Ciudad de México, provoca el crecimiento demográfico en el estado de Morelos y la migración del campo a la ciudad, el limitado empleo rural y la falta de oportunidades para la diversificación productiva de alimentos, profundiza las desigualdades que se reflejan en la marginación y la discriminación contra poblaciones campesinas e indígenas vulnerables, excluyéndolas social y culturalmente del proyecto de desarrollo estatal (Torres 2010).

En el estado de Morelos, los factores determinantes de la malnutrición social que contribuyen al hambre son: la escases de agua potable, provocada por la degradación de los ecosistemas y la pérdida de la diversidad biocultural asociada a los sistemas productivos tradicionales que incluye los huertos familiares tradicionales (HFT) y de los servicios de saneamiento.

El cambio climático global, también impacta a la agricultura local generando incertidumbre del rendimiento de los cultivos. Por lo tanto, las relaciones entre las regiones heterogéneas y el desarrollo regional homogéneo son contradictorias, ya que vulnera el ambiente y la seguridad alimentaria como resultado de la fragmentación territorial (Monroy-Ortiz 2013), cuyas consecuencias fragilizan a las comunidades humanas tanto rurales como urbanas, porque dependen de forma directa de sus bienes y de los servicios ambientales.

Una estrategia de aprovechamiento de la diversidad biocultural cultivada y silvestre, así como de los servicios agroecosistémicos son los huertos familiares tradicionales, que son unidades productivas que permiten la disponibilidad de alimentos, atributo de la seguridad alimentaria y la poliespecificidad de árboles destacando la presencia de parientes silvestres tolerados o inducidos, que les convierte en reservorios del germoplasma de las especies con valor de uso, potenciales para la seguridad alimentaria. El manejo tradicional en esta unidad productiva sintetiza los indicadores internacionales propuestos para la seguridad alimentaria:

1. La disponibilidad y estabilidad de la seguridad alimentaria se logra con la producción sostenida anual de vegetales y animales domésticos (FAO 2005), que compensa su variación en condiciones naturales por la estacionalidad climática del trópico seco mexicano.

2. El acceso y control del agua para el riego se caracteriza por escasez local, pero esta, se mitiga reusando las aguas grises del lavado de ropa, 
Ambiente y Sostenibilidad 2016 (6): 33-43

Revista del Doctorado Interinstitucional en Ciencias Ambientales

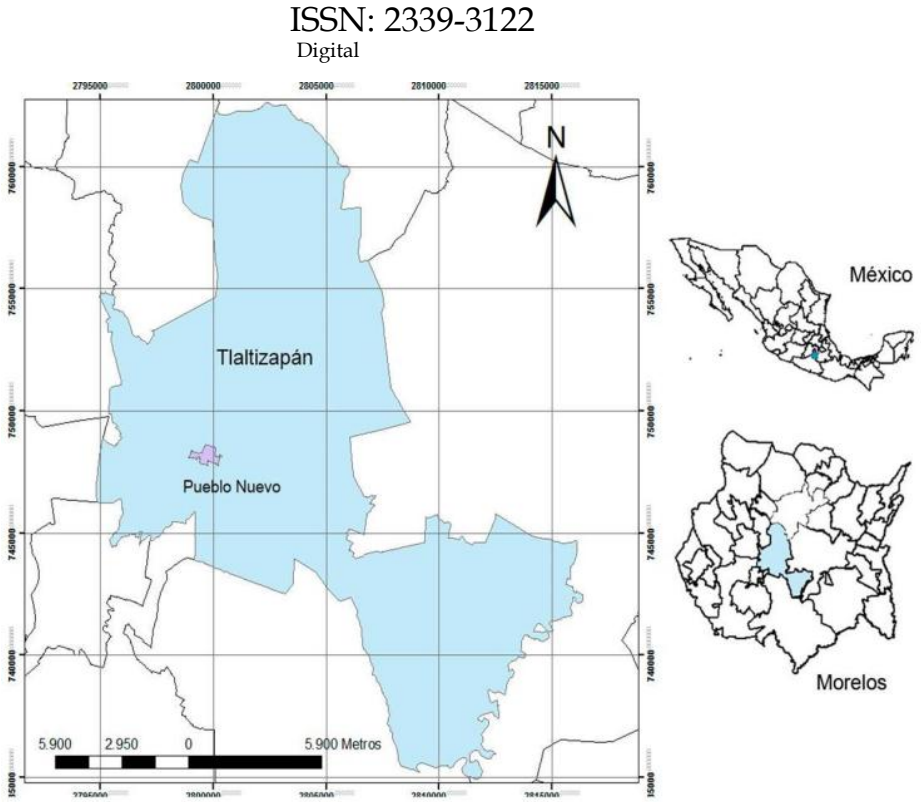

regaderas y aseo en general. Los insumos para la fertilización se obtienen del reciclaje de frutos, hojarasca, residuos orgánicos de la cocina yexcrementos de fauna domestica asociada al HFT (Colín et al. 2012).

3. El aprovechamiento o utilización cultural de la diversidad biológica se han evaluado como "la diversidad alimentaria" por Hoddinott \& Yohannes (2002) explicando su disponibilidad como fuentes de energía y proteínas.

La pregunta planteada para desarrollar este trabajo fue ¿Cuál es la importancia de los HFT para la seguridad alimentaria en las comunidades campesinas vulnerables frente a los cambios de uso del suelo?

Se parte del supuesto de que los atributos ecológicos de los HFT son producto del conocimiento, manejo y usos de la diversidad biocultural local, que se han desarrollado para satisfacer necesidades alimentarias, aunque se agregan las medicinales y energéticas, luego entonces, coinciden con los indicadores internacionales de seguridad alimentaria. El objetivo es analizar la importancia de los huertos familiares tradicionales en la seguridad alimentaria de la comunidad de Pueblo Nuevo, municipio de Tlaltizapan, Morelos, México.

\section{MATERIAL Y MÉTODOS}

\section{Área de estudio}

Pueblo Nuevo, Tlaltizapan se localiza a los $18^{\circ} 42^{\prime}$ norte y $99^{\circ} 09^{\prime}$ oeste, a una altitud sobre el nivel del mar de 970 metros, se localiza en el centro de Morelos y forma parte de la Cuenca del Río Grande Amacuzac (Figura 1).Tiene como particularidad la escasez de agua resultado del crecimiento de la zona conurbada del municipio de Cuernavaca. Junto con otras 12 comunidades conforma el consejo de pueblos, que encabeza la defensa del agua, la tierra y el aire en la región (Monroy 2009). Cuenta con una población de 1147 habitantes (INEGI 2010).

El clima es cálido con lluvias en verano, el más seco de los subhúmedos, con canícula, el porcentaje de lluvias invernal menor de cinco y marcha de la temperatura tipo Ganges, su temperatura media anual es de $23.8^{\circ} \mathrm{C}$ (Taboada et al. 2009).

Figura 1. Área de estudio.

\section{METODOLOGÍA}

El trabajo se realizó por medio de dos enfoques: el ecológico considerando el nivel de organización comunidad y el etnobotánico. En el campo, constó de dos etapas, la primera fue la prospección para ubicar los huertos. La segunda, se realizó el muestreo ecológico en las casas donde los dueños permitieron el levantamiento por medio de rectángulos de $10 \times 20$ metros en un total de 17 HFT.

En cada unidad se registró la riqueza de especies y variedades, entendidas como número total de especies y variedades. Las primeras se identificaron con claves comparando la información en documentos previos (Monroy-Ortiz \& Monroy 2006). Además, se consideró el número de individuos de cada una para calcular abundancia absoluta y relativa aplicando la siguiente fórmula:

Abundancia relativa $=\underline{\text { Suma de individuos de cada especie }} \times 100$ Abundancia absoluta

La estructura vertical del huerto se calculó con medidas dasométricas de los árboles como: cobertura, área basal $(\mathrm{DAB})$, diámetro a $1.30 \mathrm{~m}$ de altura (DAP) y altura.

Cobertura absoluta $=$ Suma de las coberturas de todas las especies

La cobertura vegetal se relacionó con servicios ambientales como la infiltración de agua.

Dominancia relativa por cobertura $=$ suma de coberturas de una especie X100

Total de coberturas de todas las especies 
Con los valores relativos de abundancia, frecuencia y dominancia relativa se obtuvo el índice de valor de importancia (IVI):

Frecuencia realtiva $=$

Número de huertos donde aparece una especieX 100

Total de huertos muestreados

$$
\begin{gathered}
\text { IVI = Abundancia relativa }+ \text { Frecuencia relativa } \\
+ \text { Dominancia relativa }
\end{gathered}
$$

Para valorar la diversificación producida por el manejo, se determinó la diversidad de los huertos por medio del Índice de diversidad Shannon-Wiener $\left(\mathrm{H}^{\prime}\right)$.

$$
\mathrm{H}^{\prime}=-\Sigma \text { pi In pi }
$$

Dónde: pi $=$ proporción (abundancia relativa) de cada especie. Fuente: Cox (1981), Stiling (1999).

Para el enfoque etnobotánico, se realizaron 17 entrevistas semiestructuradas (Galeano 2007). Esta técnica permitió conocer los aspectos de la relación cotidiana de cada propietario con el HFT, los miembros de las familias expresaron sus saberes tradicionales cuando estuvieron presentes durante el trabajo en campo, agregando una serie de hechos o ideas siempre involucrados con los recursos vegetales y animales domésticos. La información derivada se refirió al significado cultural de cada componente florístico especie y variedadcomo: nombre común, parte usada, categorías de uso, destino de la producción, época de fructificación y formas de gestión (Gispert 2010).

Un segundo aspecto, desarrollado con el propósito de explicar las relaciones con la producción de alimentos incluyó la ganadería familiar, así, se aplicaron entrevistas abiertas para obtenerlos nombres locales y el significado cultural de los animales domésticos, identificados con guías de campo. En tercer lugar se indago, el manejo comunitario, acceso y control del agua de consumo humano, por medio de 77 cuestionarios estructurados aplicados en otras visitas a los mismos informantes, además, se realizó un taller participativo. Las entrevistas incluyeron datos de los informantes como sexo, edad, escolaridad, actividad productiva principal e ingreso promedio.

\section{RESULTADOS Y DISCUSIÓN}

Los huertos familiares tradicionales son unidades productivas que cumplen algunas de las características relacionadas con la seguridad alimentaria, según el Comité de Derechos Económicos, Sociales y Culturales de las Naciones Unidas (CDESC 1999) “El derecho a la alimentación se ejerce cuando hombre, mujer o niño, dispone de alimentos sin sustancias nocivas y aceptables para una cultura determinada y la accesibilidad sostenibles. La accesibilidad comprende la económica y física".

El $65 \%$ de entrevistados fueron mujeres, el 35\% hombres encargados de podar los árboles. La edad promedio fue de 53 años, en escolaridad el 59\% solo estudio la primaria, la actividad productiva principal la desarrollan como jornaleros y obreros cuyo ingreso promedio es de $\$ 1441.00$ por semana.

\section{Enfoque Ecológico}

La gestión de las unidades productivas estudiadas se materializa de acuerdo con los resultados ecológicos de riqueza de especies y variedades en las 24 familias botánicas, que otorga la disponibilidad de bienes con 45 especies y dos variedades (Tabla 1) y en la abundancia absoluta con un valor de 274 individuos, la primera superior a la que se reporta en la localidad vecina de Acamilpa del mismo municipio, con nueve especies (Gispert et al. 2012).

Los 17 HFT muestreados en Pueblo Nuevo son poliespecíficos, de allí su alta disponibilidad alimentaria, por lo que son semejantes a los huertos de la comunidad de Xoxocotla, Morelos, (Monroy \& Colín 2012). La riqueza de especie y abundancia en los huertos fue mayor en los huertos 14 y 16 con 14 especies, 19 y 25 individuos (Figura 2). Las especies con mayor frecuencia fueron Citrus aurantifolia (limón) con 94\%, Mangifera indica (mango criollo) con $71 \%$, Spondias purpurea (ciruelo) con 65\%, Leucaena esculenta (guaje) $53 \%$ y Citrus aurantium (naranjo) con $53 \%$.

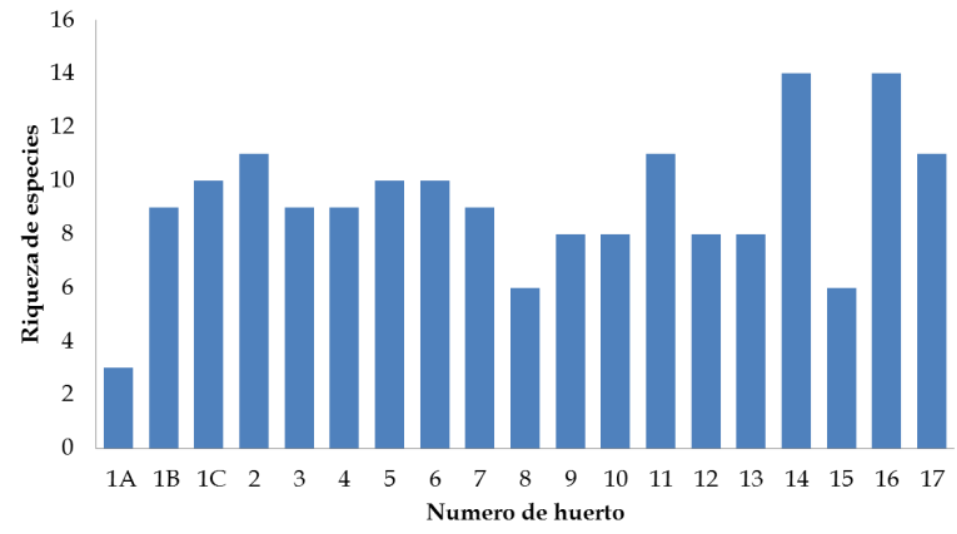

Figura 2. Poli especificidad o disponibilidad por unidad de muestreo en los huertos frutícolas tradicionales de Pueblo Nuevo, Tlaltizapan, Morelos, México. 
Ambiente y Sostenibilidad 2016 (6): 33-43

Revista del Doctorado Interinstitucional en Ciencias Ambientales

ISSN: 2339-3122

Digital

Tabla 1. Valor de índice de importancia de las especies de los huertos frutícolas tradicionales de Pueblo Nuevo, Tlaltizapan, Morelos, México. Dónde: AB.R=abundancia relativa, FRC.R= frecuencia relativa, DO.R=dominancia relativa, IVI= Valor de índice de importancia.

\begin{tabular}{|c|c|c|c|c|c|}
\hline Familia & Nombre científico & AB. R. & $\begin{array}{c}\text { FRC. } \\
\text { R. }\end{array}$ & DO. R. & IVI \\
\hline \multirow[t]{6}{*}{ Anacardiaceae } & Spondias purpurea & 8.76 & 64.70 & 1.49 & 74.95 \\
\hline & $\begin{array}{l}\text { Mangifera indica Var. } \\
\text { Manila }\end{array}$ & 1.46 & 23.53 & 0.29 & 25.28 \\
\hline & $\begin{array}{l}\text { Mangifera indica Var. } \\
\text { Petacón }\end{array}$ & 1.09 & 11.76 & 1.88 & 14.73 \\
\hline & Mangifera indica & 6.20 & 64.71 & 2.10 & 73.01 \\
\hline & Pistacia vera & 1.09 & 17.65 & 0.74 & 19.48 \\
\hline & $\begin{array}{l}\text { Amphipterygium } \\
\text { adstringens }\end{array}$ & 0.36 & 5.88 & 0.18 & 6.42 \\
\hline \multirow[t]{3}{*}{ Annonaceae } & Annona reticulata & 3.65 & 17.65 & 0.99 & 22.29 \\
\hline & Annona muricata & 1.09 & 11.76 & 1.94 & 14.79 \\
\hline & Annona diversifolia & 0.73 & 17.65 & 9.26 & 27.64 \\
\hline Apocynaceae & Plumeria rubra & 2.92 & 47.05 & 0.003 & 49.97 \\
\hline Arecaceae & Syagrus romanzoffiana & 2.92 & 17.65 & 1.84 & 22.41 \\
\hline \multirow[t]{4}{*}{ Bignoniaceae } & Parmentiera aculeata & 0.73 & 11.76 & 3.18 & 15.67 \\
\hline & Jacaranda mimosifolia & 1.09 & 11.76 & 0.35 & 13.20 \\
\hline & Pyrostegia venusta & 0.36 & 5.88 & 0.38 & 6.62 \\
\hline & $\begin{array}{l}\text { Tabebuia donnell- } \\
\text { smithii }\end{array}$ & 1.82 & 23.53 & 0.46 & 25.81 \\
\hline Boraginaceae & Ehretia tinifolia & 2.55 & 29.41 & 0.17 & 32.13 \\
\hline Burseraceae & Bursera grandifolia & 0.36 & 5.88 & 2.56 & 8.8 \\
\hline \multirow[t]{2}{*}{ Caricaceae } & Jacaratia mexicana & 0.36 & 5.88 & 3.47 & 9.71 \\
\hline & Carica papaya & 5.11 & 41.18 & 0.34 & 46.63 \\
\hline Chrysobalanaceae & Licania platypus & 0.36 & 5.88 & 0.009 & 6.24 \\
\hline Combretaceae & Terminalia catappa & 0.36 & 5.88 & 0.006 & 6.24 \\
\hline Convolvulaceae & Ipomoea arborescens & 0.36 & 5.88 & 2.07 & 8.31 \\
\hline Ebanaceae & Diospyros digyna & 1.09 & 11.76 & 8.48 & 21.33 \\
\hline \multirow[t]{2}{*}{ Euphorbiaceae } & Euphorbia fulva & 0.36 & 5.88 & 0.18 & 6.42 \\
\hline & Jatropha curcas & 1.46 & 17.65 & 1.23 & 20.34 \\
\hline \multirow[t]{5}{*}{ Fabaceae } & Leucaena esculenta & 6.57 & 41.18 & 9.56 & 57.31 \\
\hline & Pithecellobium dulce & 4.01 & 35.29 & 0.86 & 40.16 \\
\hline & Acacia farnesiana & 0.36 & 5.88 & 1.30 & 7.54 \\
\hline & Delonix regia & 1.46 & 11.76 & 2.54 & 15.76 \\
\hline & Tamarindus indica $\mathrm{L}$. & 3.28 & 41.18 & 0.15 & 44.61 \\
\hline Lauraceae & Persea americana & 4.74 & 41.18 & 0.009 & 45.92 \\
\hline Lythraceae & Punica granatum & 0.73 & 11.76 & 0.02 & 12.51 \\
\hline Malpighiacea & Byrsonima crassifolia & 0.73 & 11.76 & 0.31 & 12.80 \\
\hline Malvaceae & Ceiba pentandra & 0.36 & 5.88 & 18.34 & 24.58 \\
\hline Meliaceae & Melia azedarach & 0.36 & 5.88 & 1.1 & 7.34 \\
\hline Myrtaceae & Psidium guajava & 3.65 & 47.06 & 1.3 & 52.01 \\
\hline Oleaceae & Fraxinus uhdei & 0.36 & 5.88 & 1.06 & 7.30 \\
\hline \multirow[t]{8}{*}{ Rutaceae } & Citrus limetta & 1.09 & 5.88 & 0.87 & 7.84 \\
\hline & Citrus aurantifolia & 13.8 & 94.12 & 0.17 & 108.16 \\
\hline & & 7 & & & \\
\hline & Citrus medica & 0.36 & 5.88 & 1.25 & 7.49 \\
\hline & Citrus reticulata & 1.82 & 17.65 & 1.79 & 21.26 \\
\hline & Citrus aurantium & 5.11 & 52.94 & 0.71 & 58.76 \\
\hline & Citrus grandis & 0.36 & 5.88 & 0.08 & 6.32 \\
\hline & Casimiroa edulis & 0.36 & 5.88 & 12.11 & 18.35 \\
\hline \multirow[t]{2}{*}{ Sapotaceae } & Manilkara zapota & 2.92 & 41.18 & 2.06 & 46.16 \\
\hline & Lucuma salicifolia & 0.36 & 5.88 & 0.47 & 6.71 \\
\hline Solanaceae & Solanum erianthum & 0.36 & 5.88 & 0.23 & 6.47 \\
\hline
\end{tabular}

Los HFT tienen en su cobertura un valor que supera el área

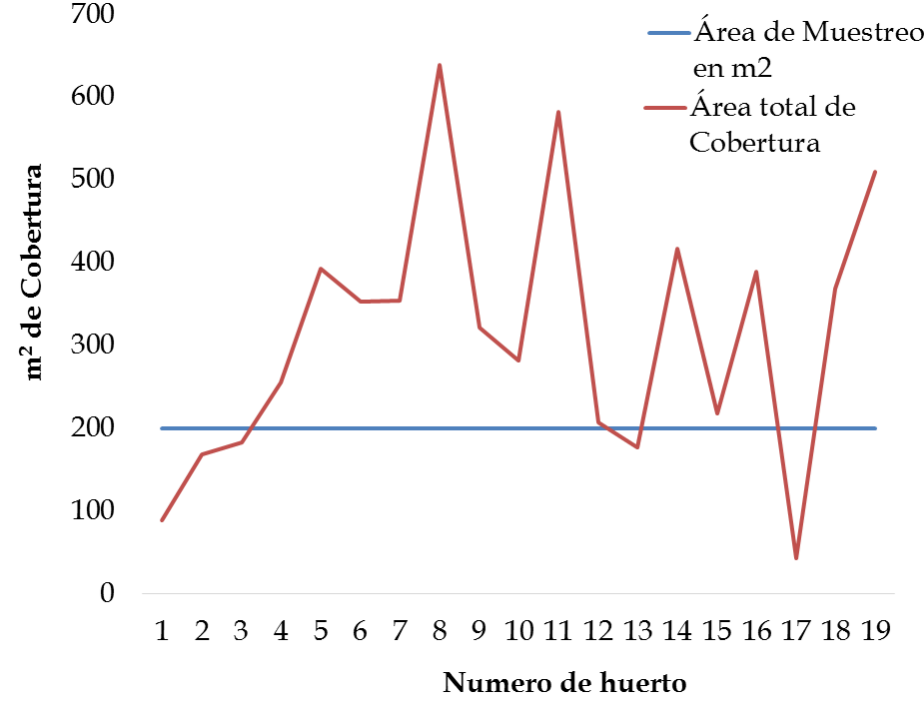

Figura 3. Relación área muestreada-cobertura en los huertos frutícolas tradicionales de Pueblo Nuevo, Tlaltizapan, Morelos, México.

El mayor valor de importancia (Tabla 1), corresponde a las especies de limón Citrus aurantifolia (IVI 108.16), ciruela Spondias purpurea (IVI 74.95), mango Mangifera indica (IVI 73.01), lo que refleja la influencia sobre los recursos espacio, agua y luz, como resultado del manejo, semejante con lo reportado por Montoya (2011) en la comunidad de Tlayacapán, Morelos, México donde las especies con mayor valor de importancia son el limón, ciruela y mango.

Los huertos de Pueblo Nuevo tienen una estructura horizontal caracterizada por la dispersión aleatoria de las especies. La variable índice de diversidad aplicada a los huertos muestreados de Pueblo Nuevo, tiene el valor de 3.29, resulta mayor del límite teórico de uno (Krebs 1978), 
mientras en otro estudio se reportan el 2.8 para Acamilpa Morelos, México y 2.1 de Bacunayagua en Cuba, (Gispert et al. 2012). Este índice se utiliza para valorar las condiciones ecológicas en el uso de los recursos que se encuentran en un espacio natural (Halfter \& Moreno 2005), pero utilizado en ambientes productivos como en este caso, explica ventajas ecológicas que el manejo otorga a las especies o variedades por razones culturales y económicas.

\section{Enfoque Etnobotánico}

Los valores de uso en orden de importancia por frecuencia de mención son: alimento, leña, sombra, medicinal cerca viva y ornamental. Esto confirma lo dicho por Pérez et al. (2012) que el diseño de los huertos en cuanto a estratificación vertical y horizontal responde a decisiones de sus propietarios para lograr la seguridad alimentaria e ingresos económicos; aportando beneficios sociales y estéticos a los hogares de las familias campesinas de todo el mundo. El origen de las especies incluye 20 (43\%) exóticasy $27(57 \%)$ nativas de la región asociadas al tipo de vegetación selva baja caducifolia, su grado de manejo va de cultivadas conel $65 \%$, toleradas con el $27 \%$ y las inducidas con $8 \%$. Otras variables que se reportan son la parte usada, el uso múltiple (Tabla 2), atributo que coincide con lo encontrado por Kantun-Balam et al. (2013) para el estado de Quintana Roo, México.

Tabla 2. Lista florística, nombre común, valor de uso, parte usada y origen de las especies. Dónde: $\mathrm{C}=$ cultivada, $\mathbf{T}=$ tolerada, $\mathbf{I}=$ inducida, $\mathbf{A l}=$ alimentaria, $\mathbf{M}=$ medicinal, $\mathbf{S}=$ sombra, $\mathbf{C v}=$ cerco vivo, Or =ornamental, L=Leña, $\mathbf{R}=$ rama,$\quad \mathbf{H}=$ hoja $\quad \mathbf{F r}=$ fruto, $\mathbf{F}=$ follaje, $\mathbf{C o}=$ corteza, $\mathbf{F l}=$ flor, $\mathbf{T}=$ tallo.

\begin{tabular}{|c|c|c|c|c|}
\hline Nombre Científico & Nombre Común & $\begin{array}{c}\text { Valor } \\
\text { de } \\
\text { uso }\end{array}$ & $\begin{array}{c}\text { Parte } \\
\text { usada }\end{array}$ & Origen \\
\hline Acacia fernesiana & Huizache & LYS & R y F & Nativa \\
\hline $\begin{array}{l}\text { Amphipterygium } \\
\text { adstringens }\end{array}$ & Cuachalalate & MyS & $\begin{array}{c}\text { Co y } \\
\text { F }\end{array}$ & Nativa \\
\hline Annona diversifolia & Ilamo & $\begin{array}{c}\mathrm{Al}, \mathrm{S} \\
\text { y L }\end{array}$ & $\begin{array}{c}\mathrm{Fr}, \mathrm{F} y \\
\mathrm{R}\end{array}$ & Nativa \\
\hline Annona muricata & Guanábana & $\begin{array}{c}\mathrm{Al}, \\
\mathrm{L}, \mathrm{S}_{\mathrm{y}} \\
\mathrm{Cv}\end{array}$ & $\begin{array}{c}\text { Fr, F y } \\
\text { R }\end{array}$ & Nativa \\
\hline Annona reticulata & Anona & $\begin{array}{c}\mathrm{Al}, \mathrm{S}, \\
\mathrm{Ly} \\
\mathrm{Cv}\end{array}$ & $\begin{array}{c}\text { Fr, F y } \\
\text { R }\end{array}$ & Nativa \\
\hline Bursera grandifolia & Palo mulato & $\begin{array}{c}\mathrm{L}, \mathrm{Cv} \\
\text { y S }\end{array}$ & $\begin{array}{c}\mathrm{R}, \mathrm{T} y \\
\mathrm{~F}\end{array}$ & Nativa \\
\hline Byrsonima crassifolia & Nanche & $\begin{array}{l}\text { Al, L } \\
\text { y S }\end{array}$ & Fr y R & Nativa \\
\hline Carica papaya & Papaya & Al y S & Fr Y F & Nativa \\
\hline Casimiro edulis & Zapote blanco & $\begin{array}{l}\mathrm{Al}, \mathrm{S} \\
\text { y L }\end{array}$ & $\begin{array}{l}\mathrm{Fr}, \mathrm{R} \\
\text { y F }\end{array}$ & Nativa \\
\hline Ceiba pentandra & Algodoncillo & $\begin{array}{c}\mathrm{L}, \mathrm{S} y \\
\mathrm{Cv}\end{array}$ & $\mathrm{R}$ & Nativa \\
\hline Citrus aurantifolia & Limón & $\mathrm{Al} \mathrm{y}$ & $\mathrm{Fr}, \mathrm{H}$ & Exótica \\
\hline
\end{tabular}

\begin{tabular}{|c|c|c|c|c|c|}
\hline \multirow{2}{*}{\multicolumn{2}{|c|}{ Citrus aurantium }} & & $\mathrm{M}$ & y Fl & \\
\hline & & Naranjo & $\begin{array}{c}\mathrm{Al} \mathrm{y} \\
\mathrm{M}\end{array}$ & Fr y H & Exótica \\
\hline \multicolumn{2}{|l|}{ Citrus grandis } & Toronja & Al y S & Fry F & Exótica \\
\hline \multicolumn{2}{|l|}{ Citrus limetta } & Lima & $\begin{array}{c}\mathrm{Al}, \mathrm{S} \\
\text { y L }\end{array}$ & Fr y R & Exótica \\
\hline \multicolumn{2}{|l|}{ Citrus medica } & $\begin{array}{l}\text { Limón mano de } \\
\text { chango }\end{array}$ & $\begin{array}{l}\mathrm{Al}, \mathrm{S} \\
\mathrm{y} \mathrm{L}\end{array}$ & $\begin{array}{l}\mathrm{Fr}, \mathrm{R} \\
\mathrm{y} \mathrm{F}\end{array}$ & Exótica \\
\hline \multicolumn{2}{|l|}{ Citrus reticulata } & Mandarina & $\begin{array}{l}\mathrm{Al}, \mathrm{S} \\
\mathrm{y} \mathrm{L}\end{array}$ & $\begin{array}{l}\mathrm{Fr}, \mathrm{R} \\
\text { y F }\end{array}$ & Exótica \\
\hline \multicolumn{2}{|l|}{ Delonix regia } & Tabachin & $\begin{array}{c}\text { Or, S } \\
\text { y L }\end{array}$ & $\begin{array}{c}\mathrm{Fl}, \mathrm{F} y \\
\mathrm{R}\end{array}$ & Exótica \\
\hline \multicolumn{2}{|l|}{ Diospyros digyna } & Zapote & $\begin{array}{c}\mathrm{Al}, \\
\mathrm{M}, \mathrm{S} \\
\text { y L }\end{array}$ & $\begin{array}{c}\text { Fr, F y } \\
\text { R }\end{array}$ & Nativa \\
\hline \multicolumn{2}{|l|}{ Ehretia tinifolia } & Palo prieto & $\begin{array}{c}\text { Or, S } \\
\text { y L }\end{array}$ & R y F & Nativa \\
\hline \multicolumn{2}{|l|}{ Euphorbia fulva } & Pega hueso & $\begin{array}{l}\text { M, L } \\
\text { y S }\end{array}$ & R y F & Nativa \\
\hline \multicolumn{2}{|l|}{ Fraxinus uhdei } & Fresno & $\begin{array}{l}\text { M, L } \\
\text { y S }\end{array}$ & R y F & Nativa \\
\hline \multicolumn{2}{|l|}{ Ipomea arborescens } & Cazahuate & $\begin{array}{l}\mathrm{L}, \mathrm{Sy} \\
\mathrm{Cv}\end{array}$ & $\begin{array}{c}\text { R, F y } \\
\text { T }\end{array}$ & Nativa \\
\hline \multicolumn{2}{|l|}{ Jacaranda mimosifolia } & Jacaranda & $\begin{array}{c}\text { O, Sy } \\
\text { L }\end{array}$ & R y F & Exótica \\
\hline \multicolumn{2}{|l|}{ Jacaratia mexicana } & Bonete & $\begin{array}{l}\mathrm{Ly} \\
\mathrm{Cv}\end{array}$ & R y T & Nativa \\
\hline \multicolumn{2}{|l|}{ Jatropha curcas } & Piñon tropical & $\mathrm{Al} \mathrm{y} \mathrm{L}$ & Fr y R & Exótica \\
\hline \multicolumn{2}{|l|}{ Leucaena esculenta } & Guaje & Al y L & Fry R & Nativa \\
\hline \multicolumn{2}{|l|}{ Licania platypus } & Zapote mechudo & $\begin{array}{l}\mathrm{Al}, \mathrm{S} \\
\text { y L }\end{array}$ & $\begin{array}{l}\text { Fr, F y } \\
\quad \mathrm{R}\end{array}$ & Exótica \\
\hline \multicolumn{2}{|l|}{ Lucuma salicifolia } & Zapote amarillo & $\begin{array}{l}\mathrm{Al}, \mathrm{S} \\
\mathrm{y} \mathrm{L}\end{array}$ & $\begin{array}{l}\text { Fr, F y } \\
\quad \mathrm{R}\end{array}$ & Nativa \\
\hline \multicolumn{2}{|l|}{ Mangifera indica $\mathrm{L}$. } & Mango criollo & $\begin{array}{l}\mathrm{Al}, \mathrm{S} \\
\mathrm{y} \mathrm{L}\end{array}$ & $\begin{array}{c}\text { Fr, F y } \\
\text { R }\end{array}$ & Exótica \\
\hline $\begin{array}{l}\text { Mangifera indica } \\
\text { Manila }\end{array}$ & Var. & Mango manila & $\begin{array}{l}\mathrm{Al}, \mathrm{S} \\
\mathrm{y} \mathrm{L}\end{array}$ & $\begin{array}{l}\text { Fr, F y } \\
\quad \mathrm{R}\end{array}$ & Exótica \\
\hline $\begin{array}{l}\text { Mangifera indica } \\
\text { Petacon }\end{array}$ & Var. & Mango petacón & $\begin{array}{l}\mathrm{Al}, \mathrm{S} \\
\mathrm{y} \mathrm{L}\end{array}$ & $\begin{array}{c}\text { Fr, F y } \\
\text { R }\end{array}$ & Exótica \\
\hline Manilkara zapota & & Chico zapote & $\begin{array}{l}\mathrm{Al}, \mathrm{S} \\
\mathrm{y} \mathrm{L}\end{array}$ & $\begin{array}{l}\text { Fr, F y } \\
\quad \mathrm{R}\end{array}$ & Nativa \\
\hline Melia azedarach & & Paraíso & $\begin{array}{l}\text { Or y } \\
\text { L }\end{array}$ & Fy R & Exótica \\
\hline Parmentiera aculeata & & Cuajilote & $\begin{array}{l}\text { M, S } \\
\text { y L }\end{array}$ & $\begin{array}{l}\text { Fr, Fy } \\
\text { R }\end{array}$ & Nativa \\
\hline Persea americana & & Aguacate & $\begin{array}{l}\mathrm{Al}, \mathrm{S} \\
\mathrm{y} \mathrm{L}\end{array}$ & $\begin{array}{l}\mathrm{Fr}, \mathrm{H} \\
\mathrm{y} \mathrm{R}\end{array}$ & Nativa \\
\hline Pistacia vera & & Pistache & $\begin{array}{l}\text { Al, L } \\
\text { y S }\end{array}$ & $\begin{array}{l}\mathrm{Fr}, \mathrm{R} \\
\text { y F }\end{array}$ & Exótica \\
\hline Pithecellobium dulce & & Huamúchil & Al y L & Fry R & Nativa \\
\hline Plumeria rubra & & Cacaloxùchil & $\begin{array}{c}\text { Or y } \\
\text { S }\end{array}$ & Fl y F & Nativa \\
\hline Psidium guajava & & Guayaba & $\begin{array}{l}\mathrm{Al}, \mathrm{M} \\
\text { y L }\end{array}$ & $\begin{array}{l}\mathrm{Fr}, \mathrm{H} \\
\mathrm{y} \mathrm{R}\end{array}$ & Nativa \\
\hline Punica granatum & & Granada & Al y L & Fry R & Exótica \\
\hline Pyrostegia venusta & & Llamarada & $\begin{array}{l}\text { Or, S } \\
\text { y L }\end{array}$ & Fy R & Exótica \\
\hline Solanum erianthum & & Quita manteca & $\begin{array}{l}\mathrm{Ly} \\
\mathrm{Cv}\end{array}$ & R y T & Nativa \\
\hline Spondias purpurea & & Ciruela & $\begin{array}{l}\mathrm{Al}, \mathrm{L} \\
\text { y S }\end{array}$ & $\begin{array}{l}\mathrm{Fr}, \mathrm{R} \\
\mathrm{y} \mathrm{F}\end{array}$ & Nativa \\
\hline Syagrus romanzoffiana & & Palma & Or & $\mathrm{F}$ & Exótica \\
\hline Tabebuia donnell-smithii & & Primavera & $\begin{array}{c}\text { Or, S } \\
\text { y L }\end{array}$ & Fy R & Nativa \\
\hline Tamarindus indica & & Tamarindo & $\begin{array}{l}\mathrm{Al}, \mathrm{S} \\
\mathrm{y} \mathrm{L}\end{array}$ & $\begin{array}{c}\text { Fr, F y } \\
\text { R }\end{array}$ & Exótica \\
\hline Terminalia catappa & & Almendro & $\mathrm{Al}$ & $\mathrm{Fr}$ & Exótica \\
\hline
\end{tabular}

La producción abastece a los miembros de las familias yel excedente se destina al mercado o intercambio, coincidiendo con Lerner (2007) quien menciona que el huerto familiar aporta una importante proporción de recursos a la economía campesina en productos con valor de uso, estos se utilizan como comestibles, ornamentales, medicinales, leña, cercos 
vivos, forrajes, ceremoniales y utensilios. Así mismo, aporta en menor proporción dinero a partir de productos con valor de cambio.

Los huertos de la comunidad tienen fructificación sostenida durante todo el año (Figura 4), aportando estabilidad porque de las 45 especies y 2 variedades, 29 son alimentarias, 11 fructifican durante junio-febrero y las 18 restantes en el periodo de sequía entre marzo-mayo. Constituyendo de esta forma como una alternativa al abasto de alimentos en áreas marginalesy complementando la dieta deficiente de sus habitantes.

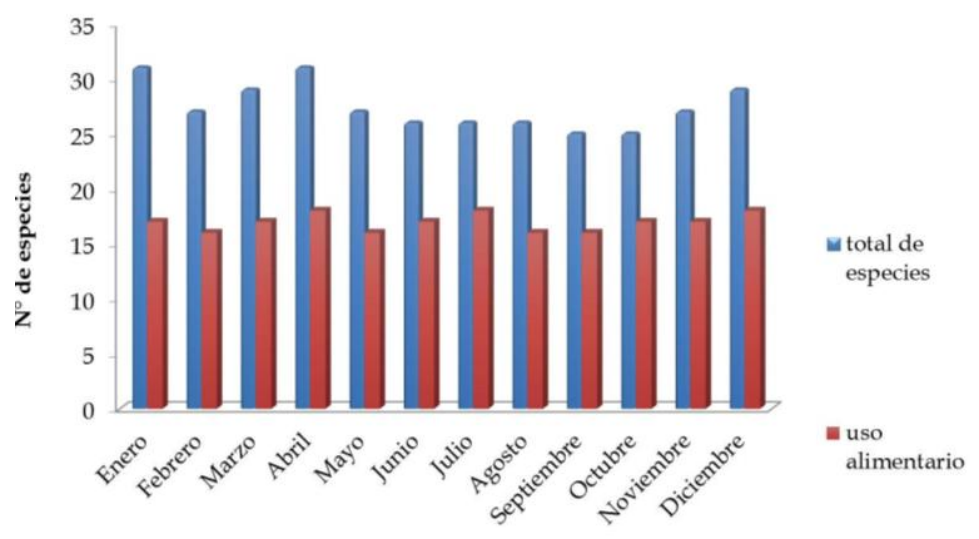

Mes de fructificación

Figura 4. Fructificación por mes de las especies de los huertos frutícolas tradicionales de Pueblo Nuevo, Tlaltizapan, Morelos, México.

Las otras 18 especies tienen usos como: ornamental, cerca viva, sombra, leña y medicinal; en esta última con ocho especies son utilizadas a pesar de la cercanía a los centros de salud, porque la población mantiene los saberes vinculados al uso de plantas curativas; según Azurdia et al. (2006), las familias asignan un alto valor a éstas porque sirven para el tratamiento de síntomas asociados a distintas enfermedades.

Las familias mantienen la tradición de cocinar en pretil (superficie en donde se coloca la estufa tradicional), utilizando la leña obtenida de las podas de los árboles de HFT, que presentan la mayor frecuencia de altura entre $1 \mathrm{y}$ 6.6 metros, seguido de 6.7 a 12.2; lo cual coincide con lo que menciona Azurdia (2008), que el producto de las podas es el material utilizado como combustible, es por eso que se muestra una mayor frecuencia para los árboles con menor altura, esto genera un ahorro en la economía de las familias, puesto que no tienen para pagar por este producto.

\section{Ganadería familiar}

En la comunidad en estudio, los animales domésticos que constituyen a la ganadería familiar (GF) o de traspatio son las gallinas (Gallus gallus), guajolotes (Meleagris gallopavo), vacas (Bos taurus), los cuales aportan carne, huevo y leche para el sustento alimentario de las familias campesinas e indígenas. El caballo (Equus ferus), tiene valor de uso como carga, para el transporte de los campesinos o de la leña que será utilizada en la cocina. La cría de animales de traspatio representa una caja de ahorro, debido a que en situaciones difíciles los animales adquieren valor de cambio (venta) para hacer frente a algunos problemas de salud, la muerte de un familiar o para adquirir útiles escolares, lo cual coincide con García y Guzmán (2014), para la comunidad Juan Nepomuceno Álvarez, municipio de Copala, Guerrero, México.

Otros animales que se localizaron en los HFT son el perro (Canis familiaris) y el gato (Felis catus) con valores de uso, protección de los hogares, compañía y control de plagas de ratones.

El arraigo de la GF se reconoce en comunidades campesinas e indígenas de México y otros países de Latinoamérica, Asia y África y su importancia radica por su contribución a resolver necesidades sociales, religiosas y el aporte de carne y huevos (Sonaiya et al. 2002). Como se ha reportado en este estudio la GF constituye un importante aporte alimentario e ingresos económicos (Medrano 2000).

La GF representauna fuente importante de aporte de proteínas para las comunidades que la practican, la cual se basa en el trabajo social de todos sus integrantes, en donde las mujeres, son quienes se encargan de la organización del huerto (Allende et al. 2012).

La especie con mayor frecuencia relativa (64.7\%) fue la gallina Gallus gallus y en menor proporción la vaca Bos taurus con el $17.4 \%$.

\section{Manejo del agua}

El reuso del agua es practicado por las familias de la comunidad, lo cual cancela el efecto de la sequía en los HFT y sostiene la reproducción de animales domésticos mitigando los problemas de la época seca del área (Jamilette2004, Jiménez 2007, Mariaca et al. 2010). Pueblo Nuevo ha logrado su autonomía en la administración del recurso agua, por medio de un Sistema Comunitario de Agua Potable (SCAP). En asambleas de usuarios del agua se discuten los problemas, los montos mensuales de cooperación, días y horas de distribución o tandeo, en la 
actualidad es cada tercer día durante tres a cinco horas, esto es una expresión de la escasez o inestabilidad.

El agua se consume sin tratamiento alguno, se distribuye por gravedad, entubada desde el manantial "Chihuahuita", al final de la ruta de abastecimiento formada por tres comunidades en orden de cercanía a la fuente principal, las localidades de Temimilcingo, Acamilpa y Pueblo Nuevo.

Las siguientes limitantes contribuyen con la escasez diferencial: las viviendas localizadas en la parte norte de la comunidad tienen mayor presión de la guaque las del sur. Además, las casas junto al apantle (canal) aprovechan el agua contaminada para el riego, vulnerando su calidad de vida.

Las formas de almacenamiento se han desarrollado en función de sus ingresos, desde pilas (tanques abiertos de tabique aplanado), tinacos, cisternas y botes. Así, entre las 17 casas muestreadas almacenan 118000 litros/año de agua. El agua es utilizada para uso doméstico incluyendo la bebida por la fauna domestica como: pollos, vacas, perros, caballos, cerdos, además de algunas especies silvestres que visitan los huertos.

Una estrategia de optimización del uso de agua frente a la escasez es reusar las aguas grises del lavado de ropa, regaderasy aseo en generalpara el riego de las plantas de los HFT. La distribución del agua para su reúso es con tuberías de pvc o por gravedad a través de canalesdirectos a cada planta, esta forma de gestión coincide con la reportada por otros autores (Aguilar 1993, Herrera-Castro 1994, Jamilette 2004, Colín et al. 2010).

El ingreso promedio per cápita es de $\$ 45.46$ diarios, menor al salario mínimo vigente que es de $\$ 63.77$. Sin embargo, el83\% de los encuestados compran agua en garrafones con costos de $\$ 10.00$ a $\$ 13.00$, el consumo promedio es de 2.6 litros/persona/día. El 17\% usan el agua de la red para beber con los riesgos de salud que esto conlleva. El 11\% captan agua de lluvia como única opción de acceso que se remonta desde los primeros asentamientos humanos, la cual, se ha dejado de aplicar debido a la tecnología.

El bajo ingreso percapita local se refleja en la falta de infraestructura para almacenar agua. Como resultado solo se almacenan $118.57 \mathrm{~m}^{3}$, de los cuales, a cada habitante le corresponden $1.53 \mathrm{~m}^{3} /$ año, de acuerdo con la UNESCO (2016), el requerimiento per cápita debe ser mayor a 500 $\mathrm{m}^{3}$ /año. Con esta referencia Pueblo Nuevo se encuentra en estado de escasez absoluta, condición que dificulta el desarrollo y producción de alimentos de la comunidad como lo ha señalado Escribano (2007).
En el taller comunitario se identificaron factores que inciden en el acceso y control del agua: 1. La construcción de la unidad habitacional "la Ciénega" sobre el área de recarga del manantial Chihuahuita. 2. El Apantle o canal que conduce el agua proveniente de dicho manantial al juntarse con el agua del Municipio de Emiliano Zapata se contamina y 3. La pérdida de un lago ubicado al norte de la comunidad que se ha secado por sobreextracción de agua (Lahera 2006).

\section{CONCLUSIONES}

La riqueza de 45 especies y dos variedades de plantas con valor de uso y tres especies de animales domésticos alimentarios, uno de carga y dos animales de compañía ejemplifica la diversidad biológica y cultural en escala comunitaria en Pueblo Nuevo, municipio de Tlaltizapan, Morelos, México. El 95.5\% de árbolesson de uso múltiple del cual, el $64 \%$ aseguran la alimentación familiar. Las especies con mayor valor de importancia ecológica corresponden a la de valor de uso alimentaria.

La escasez extrema de agua es factor esencial que agrava el hambre y la inseguridad alimentaria, la cual, se mitiga con el reuso y captación de agua, así se estabiliza la producción sostenida de los huertos derivada de la fenología de la fructificación de 29 especies alimentarias de las cuales, 11 fructifican entre junio - febrero y 18 en el periodo crítico de sequía, lo que facilita el acceso durante todo el año de alimentos nutritivos y medicinas.

En el establecimiento de los huertos familiares tradicionales se subraya que el $38 \%$ son especies silvestres toleradas y/o inducidas, incluyendo el $53 \%$ de nativas proporción importante en términos de conservación de la diversidad junto con los animales domésticos de traspatio como el guajolote Melagris gallopavo.

El manejo de la distribución espacial vertical resulta de las podas cuya leña es importante porque resuelve el acceso a combustible. La dispersión horizontal se expresa en las coberturas que van de 89 al $63 \%$ del área muestreada función que permite el aprovechamiento de la gama de los recursos disponibles e incrementa la producción de alimentos por unidad de área.

La inversión económica es mínima y satisface las necesidades diarias de las familias por medio del destino de la producciónque es para el autoabastecimiento. Son rentables porque, en su manejo social se da la transmisión oral del conocimiento tradicional de los factores bióticos y abióticos del área a través de generaciones. Esta ideología 
Ambiente y Sostenibilidad 2016 (6): 33-43

Revista del Doctorado Interinstitucional en Ciencias Ambientales

ISSN: 2339-3122

Digital

conservacionista de los campesinos e indígenas respalda la defensa de la biodiversidad territorial frente a la fragmentación y el deterioro que la política urbana produce en el Estado de Morelos, México.

\section{REFERENCIAS}

AGUILAR, L. 1993. Agroecosistemas frutícolas tradicionales de Itzamatitlán, Municipio de Yautepec, Morelos. Tesis de Licenciatura. Facultad de Ciencias Biológicas, Universidad Autónoma del Estado de Morelos,Morelos, México.

ALLENDE, N. R. JEREZ, S. M. P. VÁZQUEZ, D. M. A. \& VILLEGAS,A. Y. 2012. Estudio etno-ornitológico Auuk en Zompantle, Asunción Cacalotepec, Oaxaca: las aves de traspatio. En: VÁZQUEZ, D. M. A. \& LOPE, A. D. G. (eds). Aves y huertos de México. 48-49. Instituto Tecnológico del Valle de Oaxaca, México.

AZURDIA, C. LÓPEZ, E. OVANDO, W. \& LEIVA, M. 2006. Plantas Medicinales en Huertos Familiares. (Fecha de acceso 11 de septiembre de 2013). URL disponible en: http://www.chmguatemala.org/network/other/F11344017 43/F1160066279/F1160066527/1119222930

AZURDIA, C. 2008.Agrobiodiversidad de Guatemala. En: AZURDIA, C. GARCÍA, F. \& RÍOS, M. M. (ed). Guatemala y su biodiversidad: un enfoque histórico, cultural, biología y económico: 404-456.Serviprensa, S.A. Consejo Nacional de Áreas Protegidas. Guatemala.

BALVANERA, P. \&COTLER, H. 2009. Estado y Tendencias de los Servicios Ecosistémicos. Revista Capital Natural de México 2: 223-236.

BRAVO, M. SÁNCHEZ, J. VIDALES, J. SÁENZ, J. CHÁVEZ, J. MADRIGAL, S. MUÑOZ, H. TAPIA, L. OROZCO, G. ALCÁNTAR, J. VIDALES, I. \& VENEGAS, E. 2009. Indicadores de impacto ambiental y socioeconómico. Revista Impactos ambientales y socioeconómicos del cambio de uso del suelo forestal a huertos de aguacate en Michoacán. Instituto Nacional de Investigaciones Forestales, Agrícolas y Pecuarias 2:13-63.

COLÍN, H. HERNÁNDEZ, A. \& MONROY, R. 2010. Los huertos familiares mixtos en los altos de Morelos, México: una alternativa frente a la pobreza y escasez de agua. En: MORENO, A. PULIDO, M. T. MARICA, R. VALADEZ, R. MEJÍA, P. \& T, GUTIÉRREZ (eds). Sistemas Biocognitivos Tradicionales: paradigmas en la Conservación Biológica y el Fortalecimiento Cultural: 239-244. Asociación Etnobiológica Mexicana, A.C. México, D.F.

COLÍN, H. HERNÁNDEZ, A. \& MONROY, R. 2012. El Manejo Tradicional y Agroecológico en un Huerto Familiar de México, como ejemplo de Sostenibilidad. Revista Etnobiología, 10(2): 12-28.

COMITÉ DE DERECHOS ECONÓMICOS, SOCIALES Y CULTURALES DE LAS NACIONES UNIDAS. (CDESC). 1999. Pactointernacional de derechos económicos, sociales y culturales. (Fecha de acceso 4 de Agosto de 2015). URL disponible en: http://www.derechoshumanos.net/normativa/normas/19 66-PactoDerechosEconomicosSocialesyCulturales.htm

COX, W.G. 1981. Laboratory manual of general ecology.William C. Brown Co. Publishers. Iowa. USA. 230 pp.

ESCRIBANO, B. 2007. Una visión sostenibilista sobre la escasez del agua dulce en el mundo. Revista Internacional de Sostenibilidad, Tecnología y Humanismo. 2:85-107.

FOOD AND AGRICULTURAL ORGANIZATION (FAO). 2005. Desarrollo de las Huertas familiares. Departamento de Agricultura. Washington D.C. (Fecha de acceso 9 de enero de 2016). URL

disponible

en:http://www.fao.org/docrep/008/y5112s/y5112s05.htm

FOOD AND AGRICULTURAL ORGANIZATION FAO. 2012. Panorama de la seguridad alimentaria y nutricional en México. (Fecha de acceso 23 de Febrero de 2016). URL disponible

ftp://ftp.sagarpa.gob.mx/CGCS/Documentos/2013/Panor ama\%20Seguridad \%20Alimentaria\%20Mexico\%202012.pdf

GALEANO, M. 2007. Estrategias de investigación social cualitativa: el giro de la mirada. Medellín, Colombia. La carreta editores E.U. Pp. 29-61.

GARCÍA, F. A. \& GUZMÁN, G. E. 2014. La ganadería familiar, elemento cotidiano de los traspatios de la comunidad Juan Nepomuceno Álvarez, Copala, Guerrero, México. Sitientibus Serie Ciencias Biológicas 14: 1-11.

GISPERT, M. 2010. El proceso Cognitivo: un punto de vista Etnobotánico. En: MORENO, A. PULIDO, M. MARIACA, R. VALDEZ, R. MEJÍA, P. \& GUTIÉRREZ, T. (eds). Sistemas Biocognitivos Tradicionales, paradigma en la conservación biológica y el fortalecimiento cultural: 174-179. Asociación Etnobiologica Mexicana, A.C. México.

HALFFTER, G. \& MORENO, C. 2005. Significado biológico 
de las diversidades Alfa, Beta y Gamma. En: HALFFTER, G. SOBERÓN, J. KOLEFF, P. \& MELIC, A. (eds). Sobre diversidad biológica: el significado de las diversidades Alfa, Beta y Gamma: 5-18. SEA, CONABIO, Grupo DIVERSITAS y CONACYT, Zaragoza, España.

HERRERA-CASTRO, N. 1994. Los huertos familiares mayas en el oriente de Yucatán. Etnoflora yucatanense. Universidad Autónoma de Yucatán. Mérida, Yucatán México.169 pp.

HODDINOTT, J. \& YOHANNES, Y. 2002. Dietary Diversity as a Food Segurity indicator. Food and Nutrition Technical Assistance Project Academy for Educational Development, Washington, D.C. 47 pp.

INSTITUTO NACIONAL DE ESTADÍSTICA, GEOGRAFÍA E INFORMACIÓN (INEGI). 2010. XX Censo de Población y Vivienda.

JAMILETTE, P. 2004. Uso, reusó y reciclaje del agua residual en una vivienda. Universidad Rafael Landívar, Facultad de Ingeniería, Ingeniería Civil Administrativa, Guatemala. 64 pp.

JIMÉNEZ, W. 2007. Huertos mixtos en la economía familiar en fincas del Noratlántico de Costa Rica. Revista semestral de la Escuela de Ciencias Ambientales 33: 33-39.

KANTUN-BALAM J. FLORES J. TUN-GARRIDO J. NAVARRO-ALBERTO J. ARIAS-REYES L. \& MARTÍNEZCASILLO J. 2013. Diversidad y Origen Geográfico del Recurso Vegetal en los Huertos Familiares de Quintana Roo, México. Revista Polibotanica36:163-196.

KREBS, C. 1978. Ecología: Estudio de la Distribución y Abundancia. Harla, México, D.F. 800 pp.

LAHERA, R. 2006. Cuarto foro mundial del agua "Acciones locales para un reto global”. Comisión Nacional del Agua, World Water Council, México. 23 pp.

LERNER, T. 2007. Importancia del huerto familiar $\mathrm{Ch}^{\prime}$ ol en la economía campesina, el caso de Suclumpá, Chiapas, México. El Colegio de la Frontera Sur, Chiapas, México. 74 pp.

MARIACA, R. ÁLVAREZ, M. ARIAS, L. CAHUICH, D. GONZÁLEZ, A. VÁSQUEZ, M. \& VAN, H. 2010. Avances en el estudio de los huertos familiares del sur de México.En: MORENO, A. PULIDO, M. MARIACA, R. VALADEZ, R. MEJÍA, P. \& GUTIÉRREZ, T. (eds). Sistemas Biocognitivos tradicionales paradigmas en la Conservación Biológica y el
Fortalecimiento Cultural: 108-117. Asociación Etnobiologica Mexicana A.C. Global Diversity Foundation, Universidad Autónoma del Estado de Hidalgo, el Colegio de laFrontera Sur, Sociedad Latinoamericana de Etnobiologia, México.

MEDRANO, J. A. 2000. Recursos animales locales del centro de México. Archivos de Zootecnia 49:385-390.

MONROY, R. 2009.Problemática de las comunidades del consejo de pueblos de Morelos. En: MONROY, R. COLÍN, H. \& ROQUE-MORALES, S. (comps). Los pueblos de Morelos cabalgan por la vida: 3-9.Centro de Investigaciones Biológicas, Universidad autónoma del estado de Morelos y consejo de pueblos de Morelos. México.

MONROY, R. \& COLÍN, H. 2012. La Poliespecificidad de los Huertos Frutícolas Tradicionales. En: MONROY, R. MONROY-ORTIZ, R. \& MONROY-ORTIZ, C. (comps). Las Unidades Productivas Tradicionales: Frente a la Fragmentación Territorial: 43-56. Universidad Autónoma del Estado de Morelos, Cuernavaca Morelos, México.

MONROY-ORTIZ, C. \& MONROY, R. 2006. Las plantas, compañeras de siempre: la experiencia en Morelos. Universidad Autónoma del estado de Morelos, Cuernavaca Morelos, México. 465 pp.

MONROY-ORTIZ, R. 2013. Los sistemas urbanos de cuenca en México. Transitando a estrategias integrales de gestión hídrica. Revista Economía, Sociedad y Territorio. 13(41):151179.

MONTOYA, A. 2011. Los Huertos Tradicionales de Tlayacapan, Morelos: productores de bienes y servicios ambientales con significado cultural. Tesis de Licenciatura. Facultad de Ciencias Biológicas. Universidad Autónoma del Estado de Morelos, Cuernavaca, Morelos. México.

PÉREZ, A. CUANALO DE LA CERDA, H. \& SOLSÁNCHEZ, A. 2012. Los Huertos Familiares Perspectivas de Investigación y Contribución al Desarrollo Sustentable. En: MARIACA, R. (ed). El Huerto Familiar del Sureste de México: 131-148. ECOSUR, México.

SONAIYA, F.B. BRANCKAERT, R. D. S \& GUEYE, E. F. 2002. Research and development options for family poultry.(Fecha de acceso 12 de Julio de 2010). URL Disponible en http://www.fao.org/ag/againfo/subjects/en/infpd/econf _scope.html

STILING, P. 1999. Ecology; Theories and Applications.Prentice Hall, New Jerse, USA.840 pp. 
TABOADA, M. GRANJENO, C. A. \& OLIVER, R. 2009. Normales climatológicas (temperatura y precipitación) del estado de Morelos. Universidad Autónoma del Estado de Morelos. Cuernavaca, Morelos, México. 220 pp.

TORRES, T. F. 2010. Aspectos regionales de la Seguridad Alimentaria en México. Revista de Información y análisis. 22:1-27.

UNESCO, 2016. Informe de las Naciones Unidas sobre el desarrollo de los recursos hídricos en el Mundo. (Fecha de acceso: 11 de Marzo de 2016). URL disponible en:http://unesdoc.unesco.org/images/0024/002441/24410 3s.pdf 